\title{
Variable effect of co-infection on the HIV infectivity: Within-host dynamics and epidemiological significance
}

\author{
Diego F Cuadros ${ }^{1,2^{*}}$ and Gisela García-Ramos ${ }^{1}$
}

\author{
* Correspondence: dfc2002@qatar- \\ med.cornell.edu \\ ${ }^{1}$ Department of Biology, University \\ of Kentucky, Lexington, KY, USA \\ Full list of author information is \\ available at the end of the article
}

\begin{abstract}
Background: Recent studies have implicated viral characteristics in accounting for the variation in the HIV set-point viral load (spVL) observed among individuals. These studies have suggested that the spVL might be a heritable factor. The spVL, however, is not in an absolute equilibrium state; it is frequently perturbed by immune activations generated by co-infections, resulting in a significant amplification of the HIV viral load (VL). Here, we postulated that if the HIV replication capacity were an important determinant of the spVL, it would also determine the effect of co-infection on the VL. Then, we hypothesized that viral factors contribute to the variation of the effect of co-infection and introduce variation among individuals.
\end{abstract}

Methods: We developed a within-host deterministic differential equation model to describe the dynamics of HIV and malaria infections, and evaluated the effect of variations in the viral replicative capacity on the VL burden generated by coinfection. These variations were then evaluated at population level by implementing a between-host model in which the relationship between $V L$ and the probability of HIV transmission per sexual contact was used as the within-host and between-host interface.

Results: Our within-host results indicated that the combination of parameters generating low spVL were unable to produce a substantial increase in the VL in response to co-infection. Conversely, larger spVL were associated with substantially larger increments in the VL. In accordance, the between-host model indicated that co-infection had a negligible impact in populations where the virus had low replicative capacity, reflected in low spVL. Similarly, the impact of co-infection increased as the SpVL of the population increased.

Conclusion: Our results indicated that variations in the viral replicative capacity would influence the effect of co-infection on the VL. Therefore, viral factors could play an important role driving several virus-related processes such as the increment of the VL induced by co-infections. These results raise the possibility that biological differences could alter the effect of co-infection and underscore the importance of identifying these factors for the implementation of control interventions focused on co-infection. 


\section{Background}

At present, the role of viral genetic factors in the HIV epidemic is poorly understood. Many different HIV genetic subtypes circulate worldwide as well as their recombinant forms [1]. Moreover, there is a substantial variation in the length of the asymptomatic (chronic) phase of HIV infection, ranging from a few months to many years. This variation has been correlated with the so-called set point viral load (spVL), defined as the measure of the HIV concentration in blood during the chronic stage of the infection, representing the quasi-steady state equilibrium between virus production and clearance [1,2].

The functional differences and epidemiological significance of these variations are still unclear $[3,4]$. Several studies have indicated that host genetic factors can partially explain the variation observed in the spVL [5-7]. In addition, some other studies have revealed potential differences in the replicative capacity among different virus genotypes [8-13]. As a result, it has been suggested that if viral factors can affect the efficiency of viral replication and can be preserved from one infection to the next, this would imply a significant role of viral genetic factors determining the spVL [14].

Recent studies conducted to assess this hypothesis have evidenced that virus characteristics might indeed explain a significant proportion of the variation in the viral load (VL) observed among individuals and suggested that the spVL might be a heritable factor [15-18]. Further studies examining the HIV replicative capacity support this association between the replicative capacity of the virus and the VL $[19,20]$. These results have profound implications for important virus-related processes in which the VL is a key determinant, such as the progression, and especially in the transmission of the infection. This is given that infectiousness can be directly correlated with the concentration of HIV-RNA in plasma, which associates with shedding of the virus into genital track secretions $[21,22]$. The evidence suggests a strong correlation between the VL and HIV transmission rates [23-25]. Consequently, if viral genetic factors influence the concentration of the virus during the chronic stage of the infection, these viral characteristics might indeed play an important role on the transmission of the infection.

The spVL, however, is not in an absolute equilibrium state; it is frequently perturbed by immune activations generated by the invasion of other pathogens, resulting in a significant amplification of the VL. Changes in the host immune response induced by concomitant infections (co-infection) may account for transient increments in the VL that could make the host more infectious and increase the risk of transmission of the virus during the chronic stage of the infection [26-28]. This transient amplification of the VL generated by co-infection may be an important contributor to the successful transmission of the infection, especially in areas were the immune system is constantly challenged by multiple infections, such as in sub-Saharan Africa [29-31].

Furthermore, there is substantial heterogeneity in the effect of co-infection at the individual and population levels. Within individuals, the co-infection induced increment of the VL varies greatly [32-34]. Likewise, the effect of co-infection in the spread of the infection at the population level depends on several behavioral and epidemiological factors related to the stage and the distribution of the HIV epidemic within the population. Co-infections with sexually transmitted infections (STI) such as gonorrhea and chlamydia would have the highest impact on the transmission of the virus in populations where the epidemic is concentrated in the high risk (core) groups [35,36]. Conversely, infections prevalent in the general population such as malaria and herpes 
simplex virus type 2 (HSV-2) would be important contributors to the spread of the infection when the epidemic has invaded the general population [36,37].

Based on the biological evidence previously discussed, we suspect that viral factors might contribute to the variation in the effect of co-infection at host and population levels. Here, taking a simple modeling approach, we examined how viral factors might alter the dynamics of the virus population in the presence of co-infection and generated variation in the effects of co-infection among individuals. We expect that viruses with high replicative capacity that sustain elevated $\mathrm{spVL}$ would also generate larger transient increments in the VL in response to host immune activations induced by coinfection. In contrast, viruses with poor capacity to infect target cells and replicate would generate lower spVL and consequently lower VL increments in response of coinfection. Since the transmission efficiency of the virus among individuals is associated with the viral burden, highly infectious individuals would have viral characteristics that maximize the increment of the VL in the presence of co-infection. This result might have important implications at both the individual and the population levels.

To assess this hypothesis, we generated a mathematical model that describes the dynamics of HIV and a concomitant pathogen within the host to evaluate the variation of the viral replicative capacity on the spVL and the increment on the VL in response to co-infection. Additionally, we evaluated the effects of these variations at the population level.

\section{Methods}

We developed a deterministic differential equation compartmental model based on two independent models that describe the dynamics of HIV and a parasitic infection, namely malaria.

\section{HIV model}

The basic model of HIV dynamics implemented here has three variables: $T$ the population sizes of uninfected cells, $T^{*}$ infected cells, and $V$ free virus particles [38-40]. These quantities denote the total abundance of the given population per milliliter $(\mathrm{mL})$ of blood, and their dynamic is described by the following system of differential equations:

$$
\begin{aligned}
& \frac{d T}{d t}=s-\left(d_{t}+k_{v} V\right) T \\
& \frac{d T^{*}}{d t}=k_{v} V T-\delta T^{*} \\
& \frac{d V}{d t}=N \delta T^{*}-c V,
\end{aligned}
$$

where $s$ represents the production rate of activated immune (CD4+ T) cells, $d_{t}$ the natural death rate of activated immune cells, $k_{v}$ the HIV infection rate of activated immune cells, $\delta$ death rate of HIV infected cells, $N$ the HIV production rate by an infected cell, and $c$ the HIV removal rate. The magnitudes of these parameters are summarized in Table 1. From this system of differential equations it is possible to determine the concentration of the virus population at equilibrium, which might be 
Table 1 Co-infection within-host model parameter values

\begin{tabular}{|c|c|c|}
\hline Parameter & Value & Reference \\
\hline Rate of red blood cell production $(\Delta)$ & $2.5 \times 10^{8}$ cells $/ \mathrm{ml}$ day & {$[49,50,79]$} \\
\hline Natural death rate of uninfected red blood cells $\left(\mu_{x}\right)$ & $0.0083 /$ day & {$[46,49]$} \\
\hline Infection rate of red blood cells by merozoites $\left(k_{m}\right)$ & $\begin{array}{l}2.5 \times 10^{-10} / \text { merozoite } \\
\text { day }\end{array}$ & {$[46,49]$} \\
\hline Differentiation rate of merozoite $\left(\mu_{1}\right)$ & $0.5 /$ day & {$[46,49]$} \\
\hline Natural death rate of infected blood cells $\left(\mu_{y}\right)$ & 0.025/day & {$[46,49]$} \\
\hline $\begin{array}{l}\text { Clearance rate of infected red blood cells due to the immune } \\
\text { system }\left(\mu_{c}\right)\end{array}$ & $1 \times 10^{-8} /$ cell day & {$[50]$} \\
\hline Natural death rate of free merozoites $\left(\mu_{m}\right)$ & 48/day & {$[46,49]$} \\
\hline Death rate of merozoites by contact with immune cells $\left(\mu_{h}\right)$ & $1 \times 10^{-8} /$ cell day & {$[46,49]$} \\
\hline Merozoite production per infected red blood cell $(r)$ & 16 & {$[46,49]$} \\
\hline Production rate of immune cells (malaria specific) $(\varepsilon)$ & $1 \times 10^{-4} \mathrm{cell} / \mathrm{ml}$ day & [47] \\
\hline $\begin{array}{l}\text { Proliferation rate of immune cells in response to infected red } \\
\text { blood cells }\left(\lambda_{y}\right)\end{array}$ & $2 \times 10^{-8} /$ cell day & [50] \\
\hline Proliferation rate of immune cells in response to merozoites $\left(\lambda_{m}\right)$ & $3 \times 10^{-8} /$ merozoite day & {$[50]$} \\
\hline $\begin{array}{l}\text { Maximum number of activated immune cells per } \mathrm{ml} \text { of blood } \\
\left(T_{\max }\right)\end{array}$ & $1.5 \times 10^{6} \mathrm{cell} / \mathrm{ml}$ & [58] \\
\hline HIV infection rate for nonspecific immune cell $\left(k_{v}\right)$ & $\begin{array}{l}{\left[0.1 \times 10^{-5}-2 \times 10^{-5}\right]} \\
\text { virion/day }\end{array}$ & $\begin{array}{l}\text { Representative } \\
\text { values }\end{array}$ \\
\hline Production rate of immune cells $(s)$ & $1 \times 10^{4}$ cell/ml day & [38] \\
\hline Growth rate of non-specific immune cells $\left(r_{T}\right)$ & 0.03/day & {$[80]$} \\
\hline Natural death rate of immune cells $\left(d_{t}\right)$ & $0.01 /$ day & [81] \\
\hline Death rate of HIV infected cells $(\delta)$ & $0.7 /$ day & {$[58]$} \\
\hline HIV production by an infected cell $(N)$ & [20-300] virions/cell & $\begin{array}{l}\text { Representative } \\
\text { values }\end{array}$ \\
\hline HIV removal rate $(c)$ & 23/day & [82] \\
\hline
\end{tabular}

associated with the spVL in the chronic stage of the infection, and is described by the following equation [38]:

$$
\bar{V}=\frac{N s}{\delta c}-\frac{d_{t}}{k_{v}} .
$$

This equation shows that virus concentration at equilibrium $(\bar{V})$ may depend on virus factors that determine its replicative capacity: the infection rate of immune cells $\left(k_{v}\right)$ and the production rate by infected cells $(N)$. Since these parameters represent intrinsic characteristics of the virus, we focused on evaluating the effect of such variations on the VL burden generated by the presence of a concomitant pathogen.

\section{Malaria model}

Malaria was selected as the concomitant infection based on substantial biological and epidemiological evidence suggesting an important malaria-HIV interaction at the individual level and across regions where their incidence overlap geographically. Several studies have demonstrated the increment of VL in individuals with acute malaria $[29,32,41,42]$. Moreover, the risk of HIV infection is higher in areas with high malaria prevalence [43], and malaria has been proposed as an important facilitator for the spread of HIV in sub-Saharan Africa [36,43]. Malaria is a disease caused by protozoa of the genus Plasmodium, which are transmitted as sporozoites through bites of 
infected female Anopheles mosquitoes. During this life cycle stage, the sporozoites invade hepatocytes and replicate asexually. Each sporozoite produces thousands of merozoites per infected hepathocyte. This stage is followed by the invasion of mature red blood cells (erythrocytes) by merozoites. The rupture of an infected erythrocyte releases a few dozens of merozoite progeny that are competent to infect new erythrocytes and thus begin a new cycle $[44,45]$.

To introduce malaria parasites into the system, we implemented a model developed earlier [46] and used by several authors [47-50] to study the population dynamics of $X$ uninfected red blood cells, $Y$ infected red blood cells, $M$ free merozoites, and $T_{m}$ activated immune cells against malaria infection. This model focuses on the erythrocytic cycle of the parasite with an immunological response by the host directed against merozoite and infected red blood cells. The model assumes that the net rate of red blood cells infection is proportional to the density of uninfected red blood cells and the density population of free merozoites $\left(k_{m} M X\right)$. Likewise, the $T$ immune cell proliferation is proportional to the density of the $T$ cell and free merozoite populations $\left(\lambda_{m} M T_{m}\right)$, and the $\mathrm{T}$ cell-mediated killing of free merozoites and infected red blood cells are proportional to the density of the malaria specific immune cells $\left(\mu_{n} T_{m} M\right)$, ignoring the stimulation of B cells and other type of immune cells (processes that most probably are summarized by the density of the specific $\mathrm{T}$ cells). The system is described by the following system of differential equations:

$$
\begin{aligned}
& \frac{d X}{d t}=\Delta-\mu_{x} X-k_{m} M X \\
& \frac{d Y}{d t}=k_{m} M X-\left(\mu_{1}+\mu_{y}\right) Y-\mu_{c} Y T_{m} \\
& \frac{d M}{d t}=r \mu_{1} Y-k_{m} M X-\left(\mu_{m}+\mu_{h} T_{m}\right) M \\
& \frac{d T_{m}}{d t}=\varepsilon+\left[\lambda_{y} Y+\lambda_{m} M\right] T_{m}-d_{t} T_{m}
\end{aligned}
$$

where $\Delta$ stands for the rate of red blood cell production, $\mu_{x}$ the natural death rate of uninfected red blood cells, $k_{m}$ the infection rate of red blood cells by merozoites, $\mu_{1}$ the differentiation rate of merozoites, $\mu_{y}$ natural death rate of infected red blood cells, $\mu_{c}$ clearance rate of infected red blood cells due to the immune system, $\mu_{m}$ natural death rate of free merozoites, $\mu_{n}$ death rate of merozoites by contact with immune cells, $r$ merozoite production per infected cell, $\varepsilon$ rate of immune cell production, $\lambda_{y}$ proliferation rate of immune cells in response to infected red blood cells, and $\lambda_{m}$ proliferation rate of immune cells in response to merozoites. The parameter values are summarized in Table 1.

\section{Within-host co-infection model}

To link the within-host and between-host model, we developed a so-called nested model that connects the dynamic processes that occur at both scales. These kind of models have been previously used in epidemiology primarily to understand the pathogen evolution [51]. As previously discussed, co-infection activates host immunity, 
which in turn enhances HIV replication [27]. This phenomenon has been suggested to be a direct consequence of $\mathrm{T}$ helper cell $(\mathrm{CD} 4+\mathrm{T})$ activation that consequently raises the number of target cells susceptible to virus infection [52]. The increment in the pool of susceptible target cells has been observed in reaction to different agents that induce an immune response such as influenza vaccine [53], tetanus immunization $[33,54]$, and malaria parasites $[42,55]$. This biological argument was used to link the HIV and malaria models in a single co-infection model, and has also been used by Jones and Perelson [56] to model the effect of vaccination on chronically HIV positive persons and the effect of opportunistic infections on patients treated with antiretroviral therapy [57]. But the within-host co-infection model developed here is the first model to include the dynamics of more than one infection simultaneously to evaluate the effect of a concomitant infection on the dynamics of HIV.

It is important to note, however, that the immune stimulation that promotes HIV replication is multifactorial, and cell proliferation is only one of these mechanisms. For example, malaria infection also stimulates HIV transcription in other ways such as the activation of viral transcription via cytokines [55]. Therefore, the model presented here is a simplification of the effect of co-infection on the virus population and only describes one of the possible mechanisms in which parasites might affect the immune pathway that could alter the replication of the virus.

For the complete model of co-infection, the HIV and malaria models previously described were linked by depicting the mechanism in which malaria parasites stimulates an immune response in terms of activation and proliferation of malaria activated immune cells $\left(T_{m}\right)$, which in turn become available to HIV infection. The model includes a maximum number of immune cells per $\mathrm{mL}$ of blood $\left(T_{\max }\right)$ [58]; thus, the immune cell population will grow in a logistic fashion. After linking the two models, the dynamic of the HIVmalaria co-infection is described by the following system of differential equations:

$$
\begin{aligned}
& \frac{d X}{d t}=\Delta-\mu_{x} X-k_{m} M X \\
& \frac{d Y}{d t}=k_{m} M X-\left(\mu_{1}+\mu_{\gamma}\right) Y-\mu_{c} Y T_{m} \\
& \frac{d M}{d t}=r \mu_{1} Y-k_{m} M X-\left(\mu_{m}+\mu_{h} T_{m}\right) M \\
& \frac{d T_{m}}{d t}=\varepsilon+\left[\lambda_{y} Y+\lambda_{m} M\right]\left[1-\frac{T_{m}+T+T^{*}}{T_{\max }}\right] T_{m}-\left(d_{t}+k_{T m} V\right) T_{m} \\
& \frac{d T}{d t}=s+\left[1-\frac{T_{m}+T+T^{*}}{T_{\max }}\right] r_{T} T-\left(d_{t}+k_{v} V\right) T \\
& \frac{d T^{*}}{d t}=k_{v} V T+k_{T m} V T_{m}-\delta T^{*} \\
& \frac{d V}{d t}=N \delta T^{*}-c V .
\end{aligned}
$$


With this model, we estimated the effect of the HIV infection rate $\left(k_{v}\right)$ and production rate $(N)$ on the increment of the VL induced by co-infection by conducting several numerical simulations of the model evaluated over the bi-dimensional parameter space generated by the range parameter values of $0.1 \times 10^{-5}$ to $2 \times 10^{-5}$ /day for the HIV infection rate $\left(k_{v}\right)$, and 20 to 300 virions/cell for the HIV production rate $(N)$. We first simulated the system with HIV alone (in absence of co-infection), and then we generated several simulations with the complete system (HIV/malaria co-infection). Due to the uncertainty associated with HIV infection rate of immune cells produced by the presence of malaria parasites $\left(k_{T m}\right)$, we explored four different values for this parameter, which were proportional to the values used for the HIV infection rate $\left(k_{v}\right): k_{T m}$ $=k_{v}, k_{T m}=k_{v} / 10, k_{T m}=k_{v} / 20, k_{T m}=k_{v} / 30$. The simulations started with the introduction of HIV alone; when the dynamics of the virus reached a steady state equilibrium (the spVL), parasites were introduced into the system (day $t=600$ ). We recorded the VL immediately before the introduction of parasites, which represented the value for the spVL, and the following 30 days after the beginning of the rise of the VL generated by co-infection. The values recorded during these 30 days were then averaged to estimate the increment on the VL generated by co-infection.

To compare spVL and the increment on the VL induced by co-infection, we sampled the parameter space $\left(k_{v}, N\right)$ across several diagonal transects which generated broad combinations of the two parameters. These combinations were then used to calculate the SPVL and its corresponding increment on the VL induced by co-infection. This information was then summarized in the geometric per capita growth rate of the virus during co-infection estimated for each combination of parameters.

\section{Effect of co-infection at the population level}

Understanding how concomitant infections could influence between-host transmission and epidemic dynamics represents a major challenge in epidemiology. The scenario is even more complicated when possible viral genetic differences might introduce heterogeneity into the outcome of co-infection. For that reason, using the within-host model described previously, we attempted to evaluate the effect of virus heterogeneity on transient co-infection-induced amplification of the VL at population level.

To estimate the effect of co-infection on the spread of HIV at population level, we implemented a standard deterministic population model constructed by Abu-Raddad and coworkers [36,59]. The model is a deterministic compartmental model that stratifies the population into compartments according to HIV sero-status and stage of HIV infection, and sexual-risk activity group. The model included the three stages of HIV infection, acute, chronic and late, each one with its corresponding probability of transmission per sexual contact estimated from the literature (in the Additional file 1: Table S1). Details of the model are described elsewhere [36,59], and a short description of the model is included in the Additional file 1.

For this model, the functional relationship between VL and the probability of transmission per sexual contact was used as the within-host and between-host interface. The model assumes that the effect of the VL on the infectiousness follows the empirical relationship between VL and transmission probability per sexual contact as observed initially by Quinn and colleagues [23]: $p_{I(i) \rightarrow S(j)}^{H I V}=2.45^{\log (v l H / v l B)} p^{H I V}$, where $p_{I(i) \rightarrow S(j)}^{H I V}$ stands for the probability of transmission per sexual contact for an infected individual from the 
risk group $(i)$ to a susceptible individual from the risk group $(j), p^{H I V}$ is the baseline probability of transmission per sexual contact in the chronic stage of the infection, $v l H$ is the amplified $\mathrm{VL}$, and $v l B$ is the baseline $\mathrm{VL}$. The baseline probability of transmission per sexual contact in chronic stage was assumed to be $p^{H I V}=0.0008$, with a VL of $v l B=$ 15000 virions $/ \mathrm{mL}$ [23]. Thus, as an example of the calculations performed, at $v l H=33$ 478 virions $/ \mathrm{mL}$, the probability of transmission per sexual contact in the chronic stage is $p_{I(i) \rightarrow S(j)}^{H I V}=2.45^{\log (33478 / 15000)} \times 0.0008=0.00109$. Finally, to account for the potential effect of the VL on the HIV disease progression to AIDS, each $\log _{10}$ rise in the VL increases the rate of HIV progression to the latest stage of the infection by twofold [28].

The spVL and its corresponding co-infection induced increment on the VL previously evaluated for each combination of parameters were used to compare the spread of HIV in two different populations: one population free of co-infection, in which the probability of transmission per sexual contact during the chronic stage of the HIV infection was estimated at the spVL, and the corresponding population affected by coinfection, in which the probability of transmission during the chronic stage of the HIV infection was estimated by including the increment on the VL induced by the concomitant infection with malaria. To calculate the probability of transmission in the population affected by co-infection, it was assumed that each individual has, on average, one episode of malaria infection per year. Thus, the mean VL per year was the average of the spVL during 11 months and the increased VL for the month of the co-infection. The model was also evaluated assuming two and four episodes per year.

The system was evaluated at the endemic equilibrium (see the Additional file 1 for details). After the equilibrium solution was found through convergent successive approximations, the total incidence was calculated. Finally, to evaluate the effect of coinfection under different viral replicative capacities, the direct effect of co-infection on the HIV incidence was measured by using population attributable fractions (PAF), which is defined as the proportional reduction in disease risk over a specific time interval that can be achieved by eliminating HIV/malaria co-infection from the population $[60,61]$. It is calculated as:

$$
P A F=\left(1-\frac{I R_{v l s p}}{I R_{H v l}}\right) \times 100 \%
$$

where $I R_{v l s p}$ is the incidence rate of HIV with the spVL, and $I R_{H v l}$ is the HIV incidence rate with the heightened VL in presence of co-infection for each combination of parameters $k_{v}$ and $N$ as defined previously [36]. Due to the absence of consistent data reporting the effect of malaria-related morbidity on sexual behavior [62], we assumed that the episodes of malaria were asymptomatic as a consequence of the partial immunity generated by the consistent exposure to the parasite in areas where malaria is endemic $[63,64]$.

\section{Results}

\section{Within-host model}

The graph for the spVL (in absence of co-infection), generated in the parameter space implemented, indicated that the values for the spVL were mainly dependent on the virus replicative capacity $(N)$ (Figure 1A). In contrast, the infection rate of immune cells $\left(k_{v}\right)$ had a small effect on the estimated spVL. This result might be related to the limited number of immune cells available to be infected by the virus. Therefore, regardless of the 


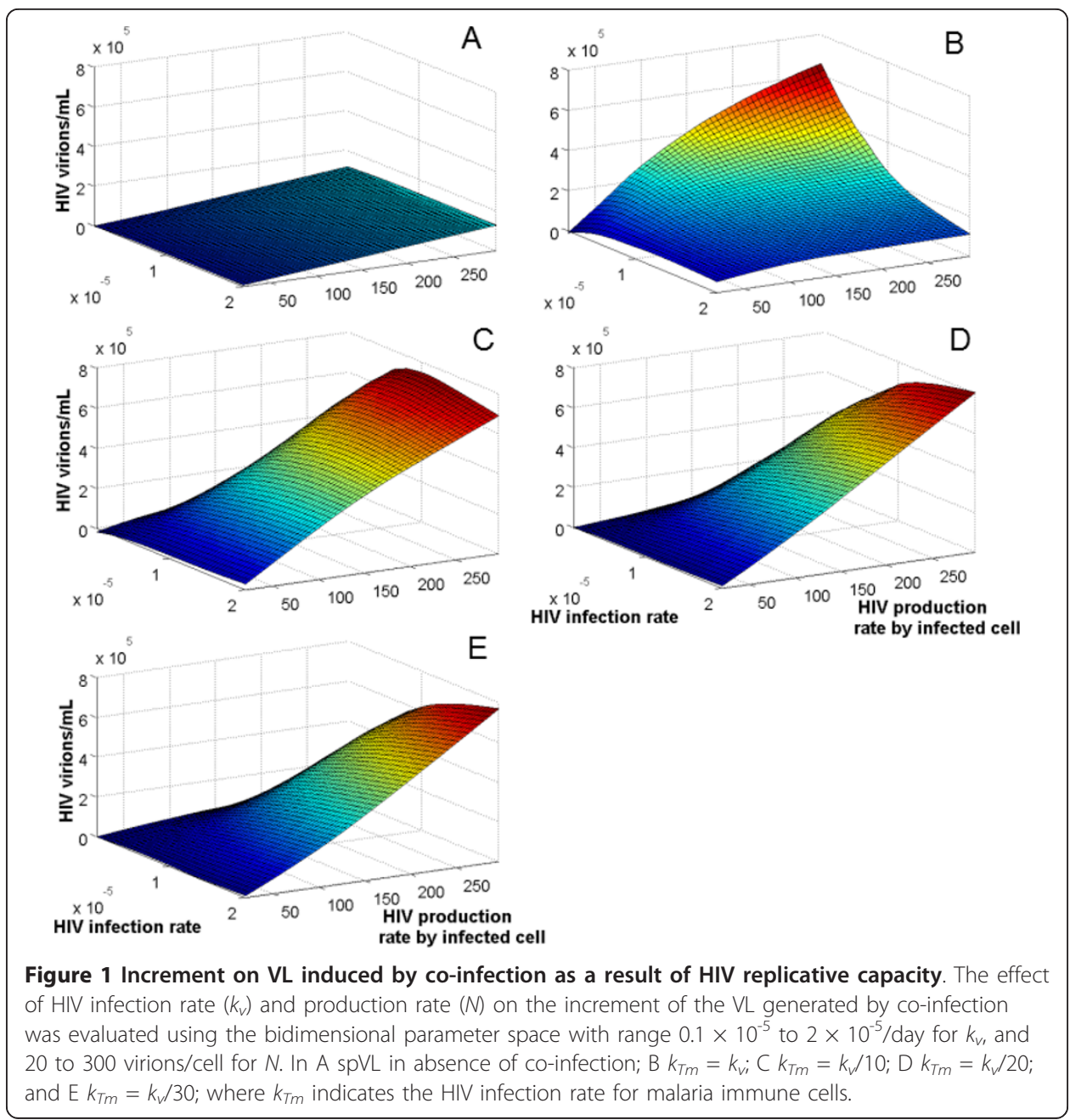

capacity of the virus to infect target cells, the spVL is limited by the number of available cells present in the system. For that reason, the number of virions produced by an infected cell $(N)$ is the main parameter that governed the estimated spVL.

On the other hand, the results from the complete system (HIV/malaria co-infection) indicated that both parameters, the infection rate of immune cells $\left(k_{v}\right)$ and the production rate by infected cells $(N)$, determine the amplification of the VL in presence of coinfection. In general, the within host co-infection model indicated that small values for the two parameters evaluated, the HIV production rate by infected cell $(N)$ and the HIV infection rate $\left(k_{v}\right)$, produced small increments on the VL when dual infection with HIV and malaria is present. Conversely, the increment on the VL increased as both parameters increased. Different values for $k_{T m}$ (HIV infection rate of immune cells for co-infection), however, generated different patterns. When $k_{T m}=k_{v}$, the differences on the increment of the VL in response to co-infection were determined by the variation on the HIV production rate per infected cell $(N)$ (Figure 1B). The highest VL amplification was produced at a very low HIV infection rate $\left(k_{v}=0.1 \times 10^{-5}\right)$ and high HIV production rate per infected cell $(N=300)$. Contrary to expectation, when $k_{v}$ increases, the increment in the VL generated by co-infection decreases. This behavior is derived from the ability of the virus to infect new target cells activated by malaria 
parasites. At high $k_{v}$, the virus rapidly infects the new target cells impeding the proliferation of these immune cells (Figure 2A). Consequently, this overexploitation of the new target cells by the virus at early stages of co-infection prevented an effective immune response to control malaria. This scenario seems to be unrealistic since individuals infected with HIV are able to have an effective immune cell proliferation in the presence of concomitant infections $[65,66]$. In addition, the increment on the VL in individuals dually infected with HIV and another pathogen is associated with an increment on the CD4+ T cells $[41,42]$.

On the other hand, when the $k_{T m}$ was a fraction of the $k_{v}$, the increment of both parameters, the HIV production rate per infected cell and the HIV infection rate, induced larger increments in the VL in response to co-infection. This result suggests that a large $k_{T m}$ might affect the availability of the new target cells for infection by the virus by diminishing the potential cell proliferation (Figure 2). When $k_{T m}=k_{v}$ l 10 , the effect of the overexploitation of target cells by the virus is still evident at high HIV infection rate (starting from $k_{v}=1 \times 10^{5}$ ) (Figure 1C). This effect is diluted when $k_{T m}=k_{v} / 20$ (Figure 1D), and the target cell concentration stabilizes with no substantial changes at smaller fractions of $k_{v}$ (Figure 1E). For that reason, and to include possible differences on the HIV infection rate generated by the availability of new target cells for HIV infection, the remaining results were derived assuming $k_{T m}=k_{v} / 20$.

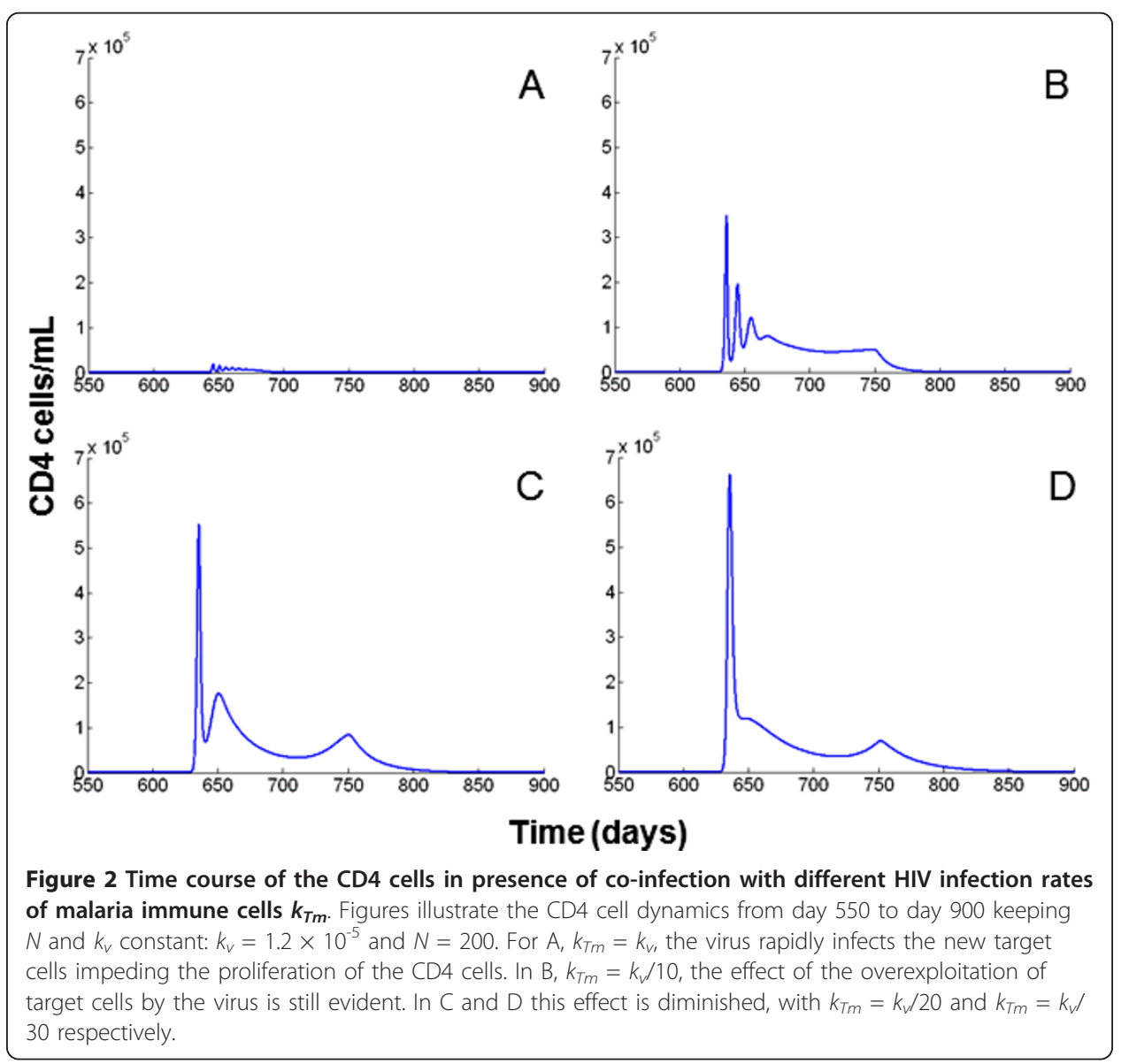


Figure 3 illustrates the temporal dynamics of the viral load according to the virus parameters used. The combination of parameters generating low spVL were almost unable to produce an increment on the VL when parasites were introduced into the system (Figure 3A, B, and 3C). Conversely, larger spVL associated with more efficient viral replicative capacity generated much larger increments in the VL in response to co-infection (Figure 3D, E, and 3F). After the introduction of parasites, there was a transient increment on virus concentration followed by a peak and successive decay to the original spVL. It is possible to observe a time delay between the introduction of the parasite and the peak of the VL (approximately 45-50 days). It might represent the time it takes for the parasite to increment its population to generate an immunological response required to perturb the VL. These viral dynamics in response to immune

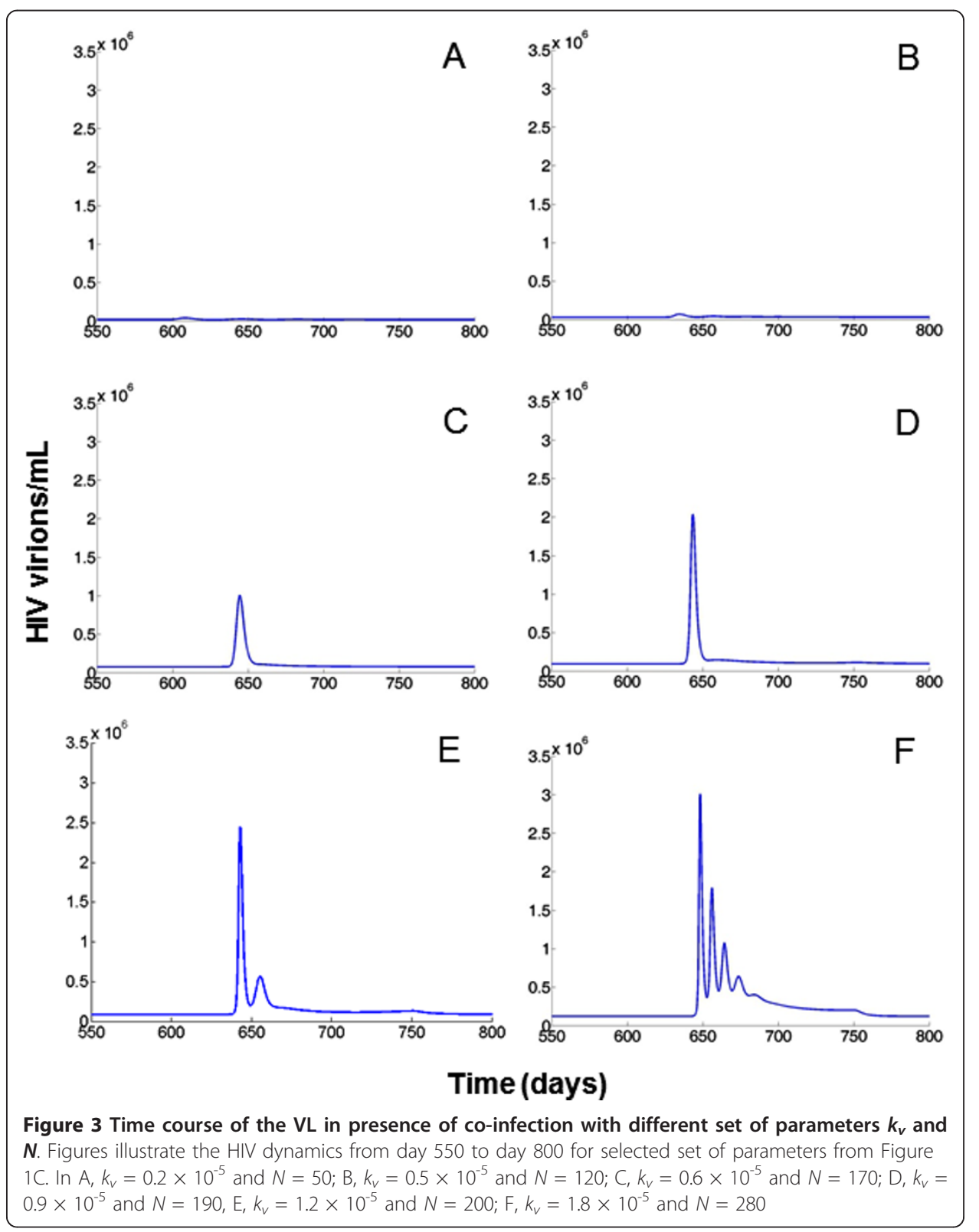


stimulations have been observed previously in vivo $[53,55]$ and replicated by other mathematical models [56,57].

On average, the VL returned to baseline levels six to eight weeks after the immune stimulation, consistent with other studies in which a large increase in the VL was observed during acute malaria and after influenza vaccine [32,53]. The average increment in the VL generated by co-infection, estimated from the 30 days recorded during the co-infection episode, ranged from 500 to 800000 virions $/ \mathrm{mL}$. This variation is consistent with the ranges estimated from studies designed to evaluate the effect of malaria on the VL of HIV-positive individuals in Africa [32,42].

The positive correlation between the spVL and the increment on the VL induced by co-infection is illustrated in Figure 4A, which was generated with the parameter values

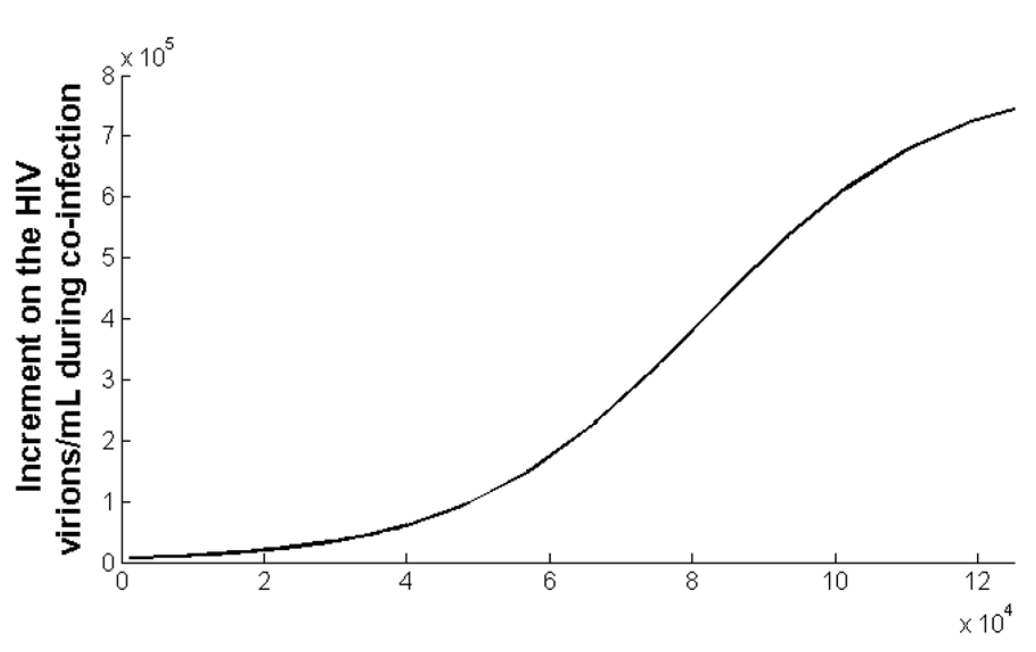

A

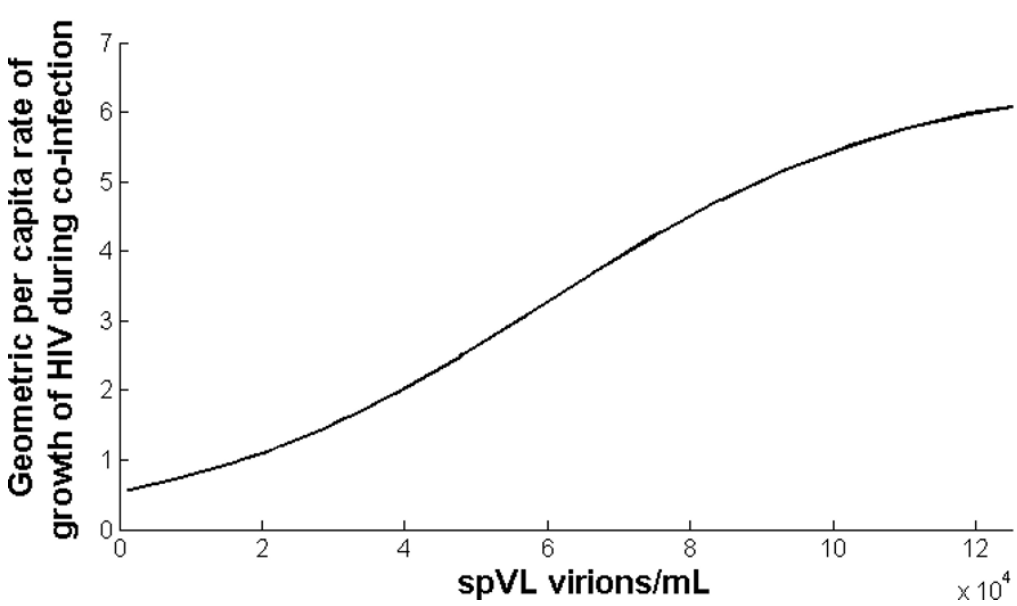

Figure 4 Correlation between the spVL and the increment on the VL in response to co-infection We used different combination of the parameters $k_{v}$ and $N$, in which both parameters increase. The values used are reported in Table S1 in the Additional file 1. In A, the results suggest that viruses with low replicative capacity conducing to low spVL are generating limited increments on the VL. Conversely, viruses with potent replicative capacity that are able to produce elevated spVL would generate much larger increments on the VL. The pattern follows a logistic fit, which suggests a saturation effect at very high spVL. In B, the geometric per capita rate of growth was estimated for each spVL, and the pattern also shows a saturation fashion. 
reported in Table S2 (see Additional file 1) for default $k_{T m}=k_{v} / 20$. Viral genotypes with poor infection rate of immune cells $\left(k_{v}\right)$ and the production rate by infected cells $(N)$, were not able to respond to increments in the activated target cells induced by the presence of concomitant infections. Consequently, the co-infection related increment in the $\mathrm{VL}$ generated by these viruses was fairly small. At lower spVL, the relationship between the increment on the virus concentration and the spVL is exponential, suggesting that viral genotypes with potent replicative capacity, and thus elevated spVL, were associated with large co-infection induced increments in the VL. The pattern followed a logistic function, suggesting a saturation effect at very high spVL (Figure 4A). Additionally, the high HIV per capita growth rate during co-infection observed at large spVL might reflect the high replication capacity of these viruses that were able to effectively respond to increments in the target cell population (Figure 4B).

\section{Between-host model}

The functional relationship between the viral load and the probability of transmission per sexual contact was used to link the viral replicative capacity and the effect of coinfection at the population level. As expected, and in accordance with the immunological model, the PAF analysis indicated that co-infection would have a negligible impact in populations where the circulating virus has low replicative capacity, reflected in low spVL. In contrast, the impact of co-infection would increase as the spVL of the population increased, and this effect levels off at around spVL $1 \times 10^{5}$ (Figure 5). This result indicates that viruses with high replicative capacity that are able to maintain elevated

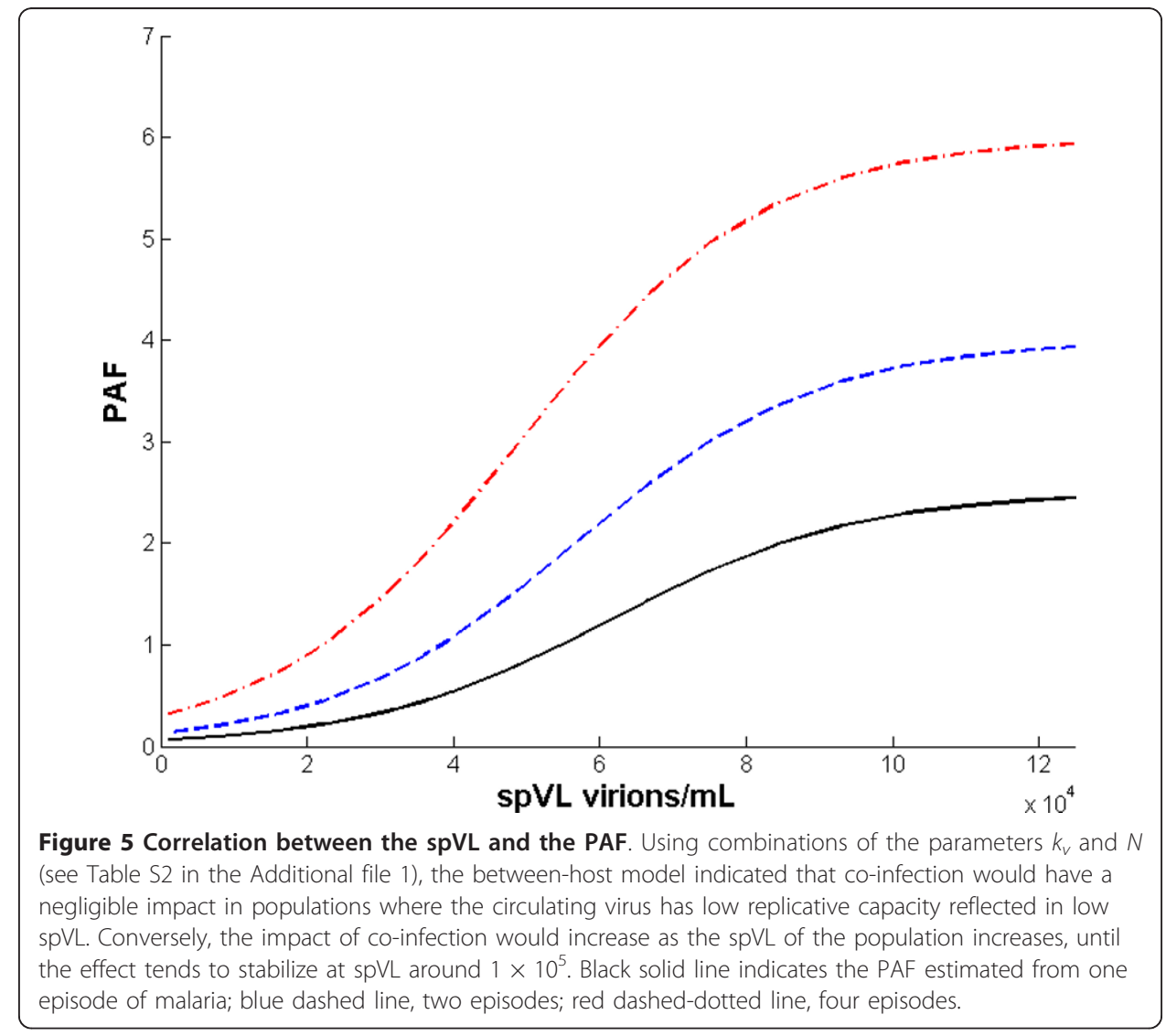


spVL would be those that have impact on the spread of the infection in terms of new HIV infections directly caused by co-infection. This result was consistent regardless of the average number of co-infection episodes per year. In addition, the number of coinfection episodes has almost no impact on the PAF at low spVL; even four episodes were not able to produce a PAF larger than $1 \%$ when the spVL is lower than $2 \times 10^{4}$.

\section{Discussion}

In this work, we explored factors that could introduce variation in the effect of coinfection on HIV dynamics at individual and population levels. We postulated that if the HIV replication capacity was an important determinant of the spVL, it would also determine the effect of co-infection on the dynamics of the virus. Here, using a previously described mathematical model to represent the single dynamics of HIV at host level $[38,39]$, it was determined that the variation in the viral replication capacity, described primarily by the HIV production by infected cell $(N)$, generated different viral load set-points. Moreover, the influence of the virus replicative capacity on the increment in VL generated by co-infection was evaluated using a within-host mathematical model of HIV/malaria co-infection which examined the effect of variation in viral infection rate of immune cells $\left(k_{v}\right)$ and the production rate by infected cells $(N)$. The model indicated that the viral replication capacity regulated by these parameters would strongly influence the induced increment of the VL when a concomitant infection is introduced in the system. In contrast to the spVL, in which the infection rate plays a minor role and is primarily determined by the production rate per infected cell, the new available target cells generated by the presence of a concomitant pathogen is a suitable condition for virus genotypes with higher infection rate of immune cells.

In addition, our model suggested the existence of a differential HIV infectivity rate for the new target cells generated by co-infection. Infection agents such as Plasmodium induce localized immune response occurring in specific organs such as the spleen [66], which produce a decrease in peripheral $\mathrm{T}$ cells as consequence of a sequestration of these cells to specific lymphoid organs [65]. These characteristics might generate a compartmentalization of the system in which not all new target cells would be available to the virus. Consequently, the HIV infection rate for the new target cells generated by co-infection $\left(k_{T m}\right.$ in our model) might be a fraction of the HIV infection rate for non-specific immune cells $\left(k_{\mathrm{v}}\right)$, as suggested by the model.

Low virus replicative capacity generated modest increments on the $\mathrm{VL}$ in response to co-infection. Regardless of the co-infection induced proliferation of target cells, the low replicative capacity prevented an effective viral response to the newly available activated target cells. Conversely, viruses with more efficient capacity to infect target cells and replicate were able to produce much stronger increments on the VL when a larger population of target cells was available. Despite the introduction of new target cells to the system, the effect of co-infection could primarily depend on the ability of the virus to efficiently exploit the temporal rise of the target cell population. This, in consequence, would generate a considerable increment on the VL when the immune system of the host is stimulated by the presence of a concomitant pathogen. This result suggests that viral factors could play an important role not only in determining the spVL but also in driving several virus-related processes such as the increment of the VL induced by co-infections. 
Our results would have important implications for the identification of highly infectious individuals. HIV-positive persons that sustain high spVL might therefore be able to produce large increments of virus concentration in response to immune stimulations and consequently become much more infectious individuals, compared to those maintaining low spVL, even in the presence of the same concomitant pathogen. This argument was then evaluated at the population level by linking the results from the within-host model with a population model using the functional relationship between viral load and probability of HIV transmission per sexual contact. We generated several virtual populations with different viral replicative capacities that sustained different mean spVL in which the direct effect of co-infection on the incidence of HIV was then evaluated. The results from these simulations were consistent with the results from the host level model and raise the possibility that co-infection might play a substantial role in the spread of HIV in populations where the circulating viruses maintain elevated spVL. Conversely, co-infection would not be an important driver of the epidemic in populations with low spVL.

This study is a theoretical exercise aimed to explore further implications of the variation of viral genetic factors on the natural history of HIV. To achieve this goal, we developed a within-host co-infection model based on two well-known and extensively used mathematical models describing the within-host dynamics of HIV and malaria infections. These two models were linked by the immune response induced by the concomitant infection with Plasmodium that increased the target cell population for HIV infection. This mechanism, however, is only one of the several mechanisms in which the invasion of another pathogen stimulates the replication of HIV $[41,53,67,68]$. The interaction between HIV and concomitant pathogens appears to be multi-factorial at cellular and immunological levels [67]. For example, the transcriptional signaling used by lymphocyte cells to regulate cell functioning is also used by HIV to regulate virus production. During co-infection, cytokines (small proteins secreted by specific cells of the immune system used for local signals between cells during response to infection [69]) might enhance their susceptibility to HIV infection and stimulate production of viruses for weeks to months without significant cytopathic effects [70,71].

Moreover, other infections might have additional mechanisms in which they could affect the transmission of HIV. For example, the genital reactivations of HSV-2 generate local immune activations in genital ulcerations that might increment HIV shedding in the genital tracks [72]. This STI infection not only affects the risk of transmission but also increases the risk of acquisition in HIV-negative individuals, a characteristic that considerably affects the mode in which HSV-2 influences the pattern of HIV transmission at the population level [36,59]. The differences in which other infections, besides malaria, interact with HIV are not considered in this study, as they may affect the results observed at the population level.

We emphasize that this work relies entirely on a hypothetical relationship between viral genotypic factors and the VL. As noted previously, regardless of the evidence about the relationship between viral genetic factors and the VL [15-17], the role of virus genotype on the natural history of HIV infection is controversial [73,74], and the evidence about differences in VL among populations is limited and very speculative $[3,75]$. Therefore, our study indicates the importance of conducting such studies that 
focus on estimating the VL to elucidate possible differences at population level. Such studies would help us better understand the extensive variation on the HIV epidemic.

Furthermore, our model does not include an evolutionary response of HIV. The virus might evolve at the two scales studied; at between-host level, the virus evolves rapidly to evade the host immune system [76]. This selection pressure favors the increment of the virus replicative capacity in the course of the infection [19]. On the other hand, at between-host level some theoretical studies have suggested that the trade-off generated between the transmission and progression of the infection implies and evolutionary stable strategy that favors virus strains with intermediate virulence [77,78]. These selection forces acting on both scales might have some important implications on the transmission efficiency of the virus that might alter the results discussed in this study. Future work would include the potential impact of these forces acting on the virus replicative capacity and the interactions with a concomitant pathogen.

Regardless of the limitations previously discussed, the model presented here highlights the possibility that viral factors might have an important impact on the role of co-infection on the spread of HIV. Therefore, understanding their variations would be a key element in the design of control interventions to prevent HIV transmission. To pursue this goal, however, it would be necessary to evaluate the results from the theoretical model proposed here with experimental data. These kinds of studies should be conducted in HIV-positive individuals in the chronic stage of the infection with similar CD4+ T cell count, and whose spVL have being well established. Their immune systems could be challenged with the application of previously used antigen immune stimulants such as tetanus toxoid vaccine [33] or influenza vaccine [34,52]. The VL should then be measured frequently (preferably daily) over a considerable period of time (at least one month), to accurately delineate the dynamics of the virus during coinfection.

As suggested by the model, biological differences could alter the effect of co-infection and underscore the importance of identifying these factors for the implementation of effective control interventions focused on co-infection. The effect of co-infection might not be the same among individuals and populations, and control strategies will not necessarily have the same impact in each population. Understanding the role of viral genetic factors on co-infection becomes an important element for developing accurate and effective recommendations for population-level control strategies.

\section{Additional material}

Additional file 1: Table S1. A single PDF file. 9 pages that include supplementary methods. The supplementary tables are embedded in the file [36,59,83-90].

Abbreviations

spVL: HIV set point viral load; VL: HIV plasma viral load; STI: Sexually transmitted infections; HSV-2: Herpes simplex virus type 2; PAF: Population attributable fraction.

Acknowledgements

We thank Phil Crowley and Susanne Awad for comments and suggestions on the model, and Maria Torres for a critical reading of the paper.

\section{Author details}

${ }^{1}$ Department of Biology, University of Kentucky, Lexington, KY, USA. ${ }^{2}$ Infectious Disease Epidemiology Group, Weill Cornell Medical College, Doha, Qatar. 


\section{Authors' contributions}

DFC wrote the draft manuscript and collaborated on project conception, model design, and simulation programming. GGR collaborated on project conception, model design and helped in writing the manuscript.

\section{Competing interests}

The authors declare that they have no competing interests.

Received: 29 November 2011 Accepted: 19 March 2012 Published: 19 March 2012

\section{References}

1. Saathoff E, Pritsch M, Geldmacher C, Hoffmann O, Koehler RN, Maboko L, Maganga L, Geis S, McCutchan FE, Kijak GH, et al: Viral and host factors associated with the hiv-1 viral load setpoint in adults from Mbeya region, Tanzania. JAIDS Journal of Acquired Immune Deficiency Syndromes 2010, 54(3):324-331.

2. Perelson A, Neumann A, Markowitz M, et al: HIV-1 dynamics in vivo: virion clearance rate, infected cell life-span, and viral generation time. Science 1996, 271:1582-1586.

3. Geretti AM: HIV-1 subtypes: epidemiology and significance fov HIV management. Current Opinion in Infectious Diseases 2006, 19(1):1-7.

4. Bonhoeffer S, Funk GA, Günthard HF, Fischer M, Müller V: Glancing behind virus load variation in HIV-1 infection. Trends in microbiology 2003, 11(11):499-504.

5. Fellay J, Shianna KV, Ge D, Colombo S, Ledergerber B, Weale M, Zhang K, Gumbs C, Castagna A, Cossarizza A, et al: A whole-genome association study of major determinants for host control of HIV-1. Science 2007, 317(5840):944-947.

6. Fellay J, Ge D, Shianna KV, Colombo S, Ledergerber B, Cirulli ET, Urban TJ, Zhang K, Gumbs CE, Smith JP, et al: Common genetic variation and the control of HIV-1 in humans. PLoS Genet 2009, 5(12):e1000791.

7. Hogan CM, Hammer SM: Host determinants in HIV infection and disease: part 2: genetic factors and implications for antiretroviral therapeutics. Annals of Internal Medicine 2001, 134(10):978-996.

8. Åsjö B, Albert J, Karlsson A, Morfeldt-Månson L, Biberfeld G, Lidman K, Fenyö E: Replicative capacity of human immunodeficiency virus from patients with varying severity of hiv infection. The Lancet 1986, 328(8508):660-662.

9. Ayele W, Mekonnen Y, Messele T, Mengistu Y, Tsegaye A, Bakker M, Berkhout B, Dorigo-Zetsma W, Wolday D, Goudsmit J, et al: Differences in HIV type 1 RNA plasma load profile of closely related cocirculating Ethiopian subtype C strains: C and C'. AIDS Res Hum Retroviruses 2010, 26(7):805-813.

10. Betts MR, Ambrozak DR, Douek DC, Bonhoeffer S, Brenchley JM, Casazza JP, Koup RA, Picker LJ: Analysis of total human immunodeficiency virus (HIV)-specific CD4+ and CD8+ T-Cell responses: relationship to viral load in untreated HIV infection. J Virol 2001, 75(24):11983-11991.

11. Blaak $H$, Brouwer $M$, Ran $L$, de Wolf $F$, Schuitemaker $H$ : In vitro replication kinetics of human immunodeficiency virus type 1 (HIV-1) variants in relation to virus load in long-term survivors of HIV-1 infection. Journal of Infectious Diseases 1998, 177(3):600-610

12. Hu DJ, Vanichseni S, Mastro TD, Raktham S, Young NL, Mock PA, Subbarao S, Parekh BS, Srisuwanvilai L, Sutthent R, et al: Viral load differences in early infection with two HIV-1 subtypes. AIDS 2001, 15(6):683-691.

13. Kivelä PS, Krol A, Salminen MO, Geskus RB, Suni Jl, Anttila V-J, Liitsola K, Zetterberg V, Miedema F, Berkhout B, et al: High plasma HIV load in the CRF01-AE outbreak among injecting drug users in Finland. Scandinavian Journal of Infectious Diseases 2005, 37(4):276-283.

14. Hollingsworth TD, Laeyendecker O, Shirreff G, Donnelly CA, Serwadda D, Wawer MJ, Kiwanuka N, Nalugoda F, CollinsonStreng A, Ssempijja V, et al: HIV-1 transmitting couples have similar viral load set-points in Rakai, Uganda. Plos Pathog 2010, 6(5):e1000876.

15. Alizon S, von Wyl V, Stadler T, Kouyos RD, Yerly S, Hirschel B, Boni J, Shah C, Klimkait T, Furrer H, et al: Phylogenetic approach reveals that virus genotype largely determines HIV set-point viral load. Plos Pathog 2010, 6(9):e1001123.

16. Fraser C, Hollingsworth TD: Interpretation of correlations in setpoint viral load in transmitting couples. AIDS 2010, 24(16):2596-2597.

17. Hecht FM, Hartogensis W, Bragg L, Bacchetti P, Atchison R, Grant R, Barbour J, Deeks SG: HIV RNA level in early infection is predicted by viral load in the transmission source. AIDS 2010, 24(7):941-945.

18. Muller V, Fraser C, Herbeck JT: A strong case for viral genetic factors in HIV virulence. Viruses 2011, 3(3):204-216.

19. Kouyos RD, von Wyl V, Hinkley T, Petropoulos CJ, Haddad M, Whitcomb JM, Boni J, Yerly S, Cellerai C, Klimkait T, et al: Assessing predicted HIV-1 replicative capacity in a clinical setting. Plos Pathog 2011, 7(11):e1002321.

20. Hinkley T, Martins J, Chappey C, Haddad M, Stawiski E, Whitcomb JM, Petropoulos CJ, Bonhoeffer S: A systems analysis of mutational effects in HIV-1 protease and reverse transcriptase. Nat Genet 2011, 43(5):487-489.

21. Attia S, Egger M, Müller M, Zwahlen M, Low N: Sexual transmission of HIV according to viral load and antiretroviral therapy: systematic review and meta-analysis. AIDS 2009, 23(11):1397-1404.

22. Baeten JM, Kahle E, Lingappa JR, Coombs RW, Delany-Moretlwe S, Nakku-Joloba E, Mugo NR, Wald A, Corey L, Donnell D, et al: Genital HIV-1 RNA predicts risk of heterosexual HIV-1 transmission. Science Translational Medicine 2011, 3(77):77-29.

23. Quinn TC, Wawer MJ, Sewankambo N, Serwadda D, Li C, Wabwire-Mangen F, Meehan MO, Lutalo T, Gray RH: Viral load and heterosexual transmission of human immunodeficiency virus type 1. New England Journal of Medicine 2000, 342(13):921-929.

24. Wilson DP, Law MG, Grulich AE, Cooper DA, Kaldor JM: Relation between HIV viral load and infectiousness: a modelbased analysis. Lancet 2008, 372(9635):314-320.

25. Hollingsworth TD, Anderson RM, Fraser C: HIV-1 transmission, by stage of infection. Journal of Infectious Diseases 2008, 198(5):687-693.

26. McLean AR, Nowak MA: Models of interactions between hiv and other pathogens. Journal of Theoretical Biology 1992, 155(1):69-102

27. Modjarrad K, Vermund SH: Effect of treating co-infections on HIV-1 viral load: a systematic review. Lancet Infectious Diseases 2010, 10(7):455-463. 
28. Modjarrad K, Chamot E, Vermund S: Impact of small reductions in plasma HIV RNA levels on the risk of heterosexual transmission and disease progression. AIDS 2008, 22:2179-2185.

29. Barnabas RV, Webb EL, Weiss HA, Wasserheit JN: The role of coinfections in HIV epidemic trajectory and positive prevention: a systematic review and meta-analysis. AIDS 2011, 25(13):1559-1573.

30. Bentwich Z, Kalinkovich A, Weisman Z: Immune activation is a dominant factor in the pathogenesis of african AIDS. Immunology Today 1995, 16(4):187-191.

31. Lawn S: AIDS in Africa: the impact of coinfections on the pathogenesis of HIV-1 infection. Journal of Infection 2004, 48(1):1-12.

32. Kublin JG, Patnaik P, Jere CS, Miller WC, Hoffman IF, Chimbiya N, Pendame R, Taylor TE, Molyneux ME: Effect of Plasmodium falciparum malaria on concentration of HIV-1-RNA in the blood of adults in rural Malawi: a prospective cohort study. Lancet 2005, 365:233-240.

33. Ostrowski MA, Krakauer DC, Li Y, Justement SJ, Learn G, Ehler LA, Stanley SK, Nowak M, Fauci AS: Effect of immune activation on the dynamics of human immunodeficiency virus replication and on the distribution of viral quasispecies. Journal of Virology 1998, 72(10):7772-7784.

34. Rosok $B$, Voltersvik $P$, Bjerknes $R$, Axelsson $M$, Haaheim LR, Asjo B: Dynamics of HIV-1 replication following influenza vaccination of HIV + individuals. Clin Exp Immunol 1996, 104(2):203-207.

35. Brunham RC, Plummer FA: A general model of sexually transmitted disease epidemiology and its implications for control. Med Clin North Am 1990, 74(6):1339-1352.

36. Abu-Raddad L, Magaret AS, Celum C, Wald A, Longini IM Jr, Self SG, Corey L: Genital herpes has played a more important role than any other sexually transmitted infection in driving HIV prevalence in Africa. PLoS One 2008, 3(5):e2230.

37. Cuadros D, Crowley P, Augustine B, Stewart S, Garcia-Ramos G: Effect of variable transmission rate on the dynamics of HIV in sub-Saharan Africa. BMC Infectious Diseases 2011, 11(1):216.

38. Callaway D, Perelson A: HIV-1 infection and low steady state viral loads. Bulletin of Mathematical Biology 2002 64(1):29-64.

39. Stafford MA, Corey L, Cao Y, Daar ES, Ho DD, Perelson AS: Modeling plasma virus concentration during primary HIV infection. Journal of Theoretical Biology 2000, 203(3):285-301.

40. Nowak M, May RM: Virus dynamics. Mathematical principles of immunology and virology New York: Oxford University Press; 2000.

41. Chen X, Xiao B, Shi W, Xu H, Gao K, Rao J, Zhang Z: Impact of acute vivax malaria on the immune system and viral load of HIV-positive subjects. Chin Med J (Engl) 2003, 116(12):1810-1820.

42. Hoffman IF, Jere CS, Taylor TE, Munthali P, Dyer JR, Wirima JJ, Rogerson SJ, Kumwenda N, Eron JJ, Fiscus SA, et al: The effect of Plasmodium falciparum malaria on HIV-1 RNA blood plasma concentration. AIDS 1999, 13(4):487-494

43. Cuadros DF, Branscum AJ, Crowley PH: HIV-malaria co-infection: effects of malaria on the prevalence of HIV in East sub-Saharan Africa. International Journal of Epidemiology 2011, 40(4):931-939.

44. Hellriegel B: Modeling the immune-response to malaria with ecological concepts - short-term behavior against long-term equilibrium. $P$ Roy Soc Lond B Bio 1992, 250(1329):249-256.

45. Malaguarnera L, Musumeci S: The immune response to Plasmodium falciparum malaria. The Lancet Infectious Diseases 2002, 2(8):472-478.

46. Anderson RM, May RM, Gupta S: Non-linear phenomena in host-parasite interactions. Parasitology 1989, 99(1):59-79.

47. Hellriegel B: Modelling the immune response to malaria with ecological concepts: short-term behaviour against long-term equilibrium. Proceedings of the Royal Society of London Series B: Biological Sciences 1992, 250(1329):249-256.

48. Mideo N, Day T, Read AF: Modelling malaria pathogenesis. Cellular Microbiology 2008, 10(10):1947-1955.

49. Tumwiine J, Mugisha JYT, Luboobi LS: On global stability of the intra-host dynamics of malaria and the immune system. Journal of Mathematical Analysis and Applications 2008, 341(2):855-869.

50. Thibodeaux J: Modeling erythropoiesis subject to malaria infection. Mathematical Biosciences 2010, 225(1):59-67.

51. Mideo N, Alizon S, Day T: Linking within- and between-host dynamics in the evolutionary epidemiology of infectious diseases. Trends in ecology \& evolution (Personal edtion) 2008, 23(9):511-517.

52. Obrien WA, Grovitferbas K, Namazi A, Ovcakderzic S, Wang HJ, Park J, Yeramian C, Mao SH, Zack JA: Human immunodeficiency virus-type-1 replication can be increased in peripheral-blood of seropositive patients after influenza vaccination. Blood 1995, 86(3):1082-1089.

53. Staprans SI, Hamilton BL, Follansbee SE, Elbeik T, Barbosa P, Grant RM, Feinberg MB: Activation of virus replication after vaccination of HIV-1-infected individuals. J Exp Med 1995, 182(6):1727-1737.

54. Cheynier R, Henrichwark S, Hadida F, Pelletier E, Oksenhendler E, Autran B, Wainhobson S: Hiv and T-Cell expansion in splenic white pulps is accompanied by infiltration of HIV-specific cytotoxic t-lymphocytes. Cell 1994, 78(3):373-387.

55. Xiao L, Owen S, Rudolph D, Lal R, Lal A: Plasmodium falciparum antigen induced human inmmunodeficiency virus type I replication is mediated trhough induction of tumor necrosis factor. The Journal of Infectious Diseases 1998, 117:437-445.

56. Jones LE, Perelson AS: Modeling the effects of vaccination on chronically infected HIV-positive patients. JAIDS Journal of Acquired Immune Deficiency Syndromes 2002, 31(4):369-377.

57. Jones L, Perelson A: Opportunistic infection as a cause of transient viremia in chronically infected HIV patients under treatment with HAART. Bulletin of Mathematical Biology 2005, 67(6):1227-1251.

58. Perelson AS, Kirschner DE, De Boer R: Dynamics of HIV infection of CD4+ T cells. Mathematical Biosciences 1993, 114(1):81-125.

59. Abu-Raddad LJ, Patnaik P, Kublin JG: Dual infection with HIV and malaria fuels the spread of both diseases in subSaharan Africa. Science 2006, 314(5805):1603-1606.

60. Rockhill B, Newman B, Weinberg C: Use and misuse of population attributable fractions. American Journal of Public Health 1998, 88(1):15-19.

61. Levine B: What does the population attributable fraction mean? Preventing chronic disease 2007, 4(1):A14.

62. Van geertruyden J-P, D'Alessandro U: Malaria and HIV: a silent alliance. Trends in Parasitology 2007, 23(10):465-467. 
63. Geertruyden J-PV, Mulenga M, Kasongo W, Polman K, Colebunders R, Kestens L, D'Alessandro U: CD4 T-Cell count and HIV-1 infection in adults with Uncomplicated Malaria. JAIDS Journal of Acquired Immune Deficiency Syndromes 2006, 43(3):363-367.

64. Kublin JG, Patnaik P, Jere CS, Miller WC, Hoffman IF, Chimbiya N, Pendame R, Taylor TE, Molyneux ME: Effect of Plasmodium falciparum malaria on concentration of HIV-1-RNA in the blood of adults in rural Malawi: a prospective cohort study. Lancet 2005, 365(9455):233-240.

65. Wyler DJ: Peripheral lymphocyte subpopulations in human falciparum malaria. Clinical \& Experimental Immunology 1976, 23(3):471-476.

66. Chougnet $C$, Tallet $S$, Ringwald P, Deloron P: Kinetics of lymphocyte subsets from peripheral blood during a Plasmodium falciparum malaria attack. Clinical \& Experimental Immunology 1992, 90(3):405-408.

67. Copeland KF, Heeney JL: T helper cell activation and human retroviral pathogenesis. Microbiological reviews 1996, 60(4):722-742.

68. Pisel T, Hoffman I, Jere C, et al: Immune activation and induction of HIV-1 replication within CD14 macrophages during acute Plasmodium falciparum malaria coinfection. AIDS 2002, 16:1503-1509.

69. Graham AL, Cattadori IM, Lloyd-Smith JO, Ferrari MJ, Bjørnstad ON: Transmission consequences of coinfection: cytokines writ large? Trends in Parasitology 2007, 23(6):284-291.

70. Kedzierska K, Crowe S, Turville S, Cunningham A: The influence of cytokines, chemokines and their receptors on HIV1 replication in monocytes and macrophages. Rev Med Virol 2003, 13:39-56.

71. Vicenzi E, Biswas P, Mengozzi M, Poli G: Role of pro-inflammatory cytokines and beta-chemokines in controlling HIV replication. J Leukocyte Biol 1997, 62:34-40.

72. McClelland RS, Wang CC, Overbaugh J, Richardson BA, Corey L, Ashley RL, Mandaliya K, Ndinya-Achola J, Bwayo JJ, Kreiss JK: Association between cervical shedding of herpes simplex virus and HIV-1. AIDS 2002, 16(18):2425-2430

73. Hemelaar J, Gouws E, Ghys PD, Osmanov S: Global and regional distribution of HIV-1 genetic subtypes and recombinants in 2004. AIDS 2006, 20(16):W13-W23.

74. Thomson MM, Perez-Alvarez L, Najera R: Molecular epidemiology of HIV-1 genetic forms and its significance for vaccine development and therapy. Lancet Infectious Diseases 2002, 2(8):461-471.

75. Morison L, Buve A, Zekeng L, Heyndrickx L, Anagonou S, Musonda R, Kahindo M, Weiss HA, Hayes RJ, Laga M, et al: HIV-1 subtypes and the HIV epidemics in four cities in sub-Saharan Africa. AIDS 2001, 15:S109-S116.

76. Leslie AJ, Pfafferott K, Chetty P, Draenert R, Addo MM, Feeney M, Tang Y, Holmes EC, Allen T, Prado JG, et al: HIV evolution: CTL escape mutation and reversion after transmission. Nat Med 2004, 10(3):282-289.

77. Fraser C, Hollingsworth TD, Chapman R, de Wolf F, Hanage WP: Variation in HIV-1 set-point viral load: Epidemiological analysis and an evolutionary hypothesis. Proceedings of the National Academy of Sciences 2007 104(44):17441-17446.

78. Shirreff $G$, Pellis $L$, Laeyendecker $O$, Fraser $C$ : Transmission selects for HIV-1 strains of intermediate virulence: a modelling approach. PLoS Comput Biol 2011, 7(10):e1002185.

79. SAUL A: Parasitology 1998, 117(05):405-407.

80. Perelson AS, Kirschner DE, De Boer R: Dynamics of HIV infection of CD4+ T cells. Math Biosci 1993, 114(1):81-125.

81. Mohri H, Bonhoeffer S, Monard S, Perelson AS, Ho DD: Rapid turnover of T lymphocytes in SIV-infected Rhesus macaques. Science 1998, 279(5354):1223-1227.

82. Ramratnam B, Bonhoeffer S, Binley J, Hurley A, Zhang L, Mittler JE, Markowitz M, Moore JP, Perelson AS, Ho DD: Rapid production and clearance of HIV-1 and hepatitis C virus assessed by large volume plasma apheresis. The Lancet 1999, 354(9192):1782-1785.

83. Wawer MJ, Gray RH, Sewankambo NK, Serwadda D, Li XB, Laeyendecker O, Kiwanuka N, Kigozi G, Kiddugavu M, Lutalo T, et al: Rates of HIV-1 transmission per coital act, by stage of HIV-1 infection, in Rakai, Uganda. Journal of Infectious Diseases 2005, 191(9):1403-1409.

84. Garnett GP, Anderson RM: Factors controlling the spread of hiv in heterosexual communities in developing countries: patterns of mixing between different age and sexual activity classes. Philosophical Transactions: Biological Sciences 1993, 342(1300):137-159.

85. Hollingsworth TD, Anderson RM, Fraser C: HIV-1 transmission, by stage of infection. Journal of Infectious Diseases 2008, 198(5):687-693.

86. Pinkerton S: Probability of HIV transmission during acute infection in Rakai, Uganda. AIDS and Behavior 2008, 12(5):677-684

87. Todd J, Glynn JR, Marston M, Lutalo T, Biraro S, Mwita W, Suriyanon V, Rangsin R, Nelson KE, Sonnenberg P, et al: Time from HIV seroconversion to death: a collaborative analysis of eight studies in six low and middle-income countries before highly active antiretroviral therapy. AIDS 2007, 21:S55-S63.

88. Ferry B, Caraël M, Buvé A, Auvert B, Laourou M, Kanhonou L, de Loenzien M, Akam E, Chege J, Kaona F: Cities $\mathrm{ftSGoHoHEiA:} \mathrm{Comparison} \mathrm{of} \mathrm{key} \mathrm{parameters} \mathrm{of} \mathrm{sexual} \mathrm{behaviour} \mathrm{in} \mathrm{four} \mathrm{African} \mathrm{urban} \mathrm{populations} \mathrm{with} \mathrm{different}$ levels of HIV infection. AIDS 2001, 15:S41-S50.

89. Morison L, Weiss HA, Buvé A, Caraël M, Abega S-C, Kaona F, Kanhonou L, Chege J, Hayes RJ: Cities ftSGoHoHEiA: Commercial sex and the spread of HIV in four cities in sub-Saharan Africa. AIDS 2001, 15:S61-S69.

90. Buve A, Weiss HA, Laga M, Van Dyck E, Musonda R, Zekeng L, Kahindo M, Anagonou S, Morison L, Robinson NJ, et al: The epidemiology of gonorrhoea, chlamydial infection and syphilis in four African cities. Aids 2001, 15:S79-S88.

\section{doi:10.1186/1742-4682-9-9}

Cite this article as: Cuadros and García-Ramos: Variable effect of co-infection on the HIV infectivity: Within-host dynamics and epidemiological significance. Theoretical Biology and Medical Modelling 2012 9:9. 